\title{
DUST IN COMETARY COMAE: PRESENT UNDERSTANDING OF THE STRUCTURE AND COMPOSITION OF DUST PARTICLES
}

\author{
A.C. LEVASSEUR-REGOURD \\ Université Pierre et Marie Curie-Paris6, UMR 7620, Paris, F-75005; \\ CNRS, Service d'Aéronomie, BP3, 91371 Verrières, France

\section{ZOLENSKY} \\ KT NASA Johnson Space Center, \\ Houston, TX 77058 US \\ J. LASUE \\ Université Pierre et Marie Curie-Paris6, UMR 7620, Paris, F-75005; \\ CNRS, Service d'Aéronomie, BP3, 91371 Verrières, France
}

\begin{abstract}
In situ probing of a very few cometary comae has shown that dust particles present a low albedo and a low density, and that they consist of both rocky material and refractory organics. Remote observations of solar light scattered by cometary dust provide information on the properties of dust particles in the coma of a larger set of comets. The observations of the linear polarization in the coma indicate that the dust particles are irregular, with a size greater (on the average) than about one micron. Besides, they suggest, through numerical and experimental simulations, that both compact grains and fluffy aggregates (with a power law of the size distribution in the -2.6 to -3 range), and both rather transparent silicates and absorbing organics are present in the coma. Recent analysis of the cometary dust samples collected by the Stardust mission provide a unique ground truth and confirm, for comet $81 \mathrm{P} /$ Wild 2, the results from remote sensing observations. Future space missions to comets should, in the next decade, lead to a more precise characterization of the structure and composition of cometary dust particles.
\end{abstract}

\section{Relevance of cometary dust studies}

Whenever the solid part of a comet, i.e. its nucleus of ices and dust, passes close to the Sun on an elongated orbit, some of its ices sublimate. The released gases carry out solid particles, and form with them a bright coma of gas and dust. 
Understanding the structure and composition of dust particles in cometary comae provides information on the cometary environment (mandatory for the success of cometary missions, e.g. Rosetta ${ }^{1}$ ) and on the contribution of cometary dust to the zodiacal cloud (interplanetary dust cloud, mostly replenished by dust ejected from cometary nuclei and released by asteroidal collisions ${ }^{2,3}$ ). Moreover, it provides clues to the processes that were leading to the formation of cometary nuclei in the early solar system, and to the role of their impacts in the evolution of the early solar system. These studies are all the more significant, since cometary nuclei are probably the least-altered objects surviving from the origin of the solar system. Detailed reviews on the current knowledge about comets can be found in the Comets II book ${ }^{4}$.

It is likely that, in the protosolar nebula, low-velocity collisions of sub micron grains allowed them to stick together by van der Waals forces and lead to a rapid growth of fluffy fractal dust agglomerates ${ }^{5,6}$, with fractal dimensions below 2. Macroscopic aggregates could then progressively be formed, although it should be added that the mutual interactions between icy and refractory grains are not yet fully understood ${ }^{7}$.

In this paper, we present direct information on dust in cometary comae obtained from past in situ missions and from collections of interplanetary dust particles collected in the Earth stratosphere (section 2). Then, we discuss the significance of remote studies extensively performed during the past decades, with emphasis on light scattering data and their interpretation (section 3). An overview of the evidence provided by recent in situ missions, e.g. Stardust samples analysis, is given in section 4 . We conclude with perspectives expected from future missions in section 5 .

\section{Evidence from past in situ studies}

More than 20 years ago, dust instruments flying-by comet 1P/Halley on board Vega and Giotto space probes have discovered the presence of carbonaceous material in cometary dust, with both rocky particles, e.g. silicates, metals, sulphides with $\mathrm{Mg}, \mathrm{Si}, \mathrm{Ca}, \mathrm{Fe}$, and so-called "CHON" particles, i.e. refractory organics such as polymerized formaldehyde and polyoxymethylene $e^{8,9}$. Carbonaceous and rocky materials might be mixed together on a very fine scale, and have a mass ratio - in the coma - of about 1.

With a miss distance of about $600 \mathrm{~km}$, the Giotto instruments have confirmed that the dust coma is highly structured and heterogeneous (with e.g. evidence for jet-like features and changes in the local polarization) and that its size-range spans from submicron-sized particles to large, millimetre-sized particles. They have also allowed us, from a comparison of light scattering and 
dust flux data ${ }^{10}$, to estimate that the dust has a low albedo (of about 0.04), that its size distribution approximately follows a power law (such as $\mathrm{s}^{-2.6 \pm 0.2}$ ), and that its density (of about $100 \mathrm{~kg} \mathrm{~m}^{-3}$ ), is extremely low ${ }^{11}$.

Later on, the flyby of comet 26P/Grigg-Skjellerup by Giotto (with a missdistance in the 150-200 km range) has led to the detection of an asymmetry in the coma, possibly induced by an active fragment in the $10 \mathrm{~m}$ size range ${ }^{12}$. The hypothesis of a possible fragmentation, and thus of a significant fragility of the nuclei, has soon been strengthened by remote observations of numerous fragmentations and even total disruptions of nuclei. The tidal disruption of D/1993 F2 Shoemaker-Levy 9 (which impacted Jupiter in July 1994) and the successive fragmentations of 73P/Schwassmann-Wachmann 3 from 1996 to 2006 provide typical examples of this fragility.

The composition of cometary dust has also been studied through laboratory analyses of IDPs, i.e. interplanetary dust particles collected in the Earth stratosphere after they have suffered atmospheric entry ${ }^{2}$. Some anhydrous chondritic IDPs, actually fragile and highly porous aggregates (Fig. 1a), are suspected to be of cometary origin. Their analysis ${ }^{13,14}$ reveals an un-equilibrated mixture of both so-called GEMS (for glass with embedded metal and sulfides), which could be radiation-damaged residues of early-formed solar system materials, and high temperature condensates of crystalline silicates (e.g. forsterite and enstatite).

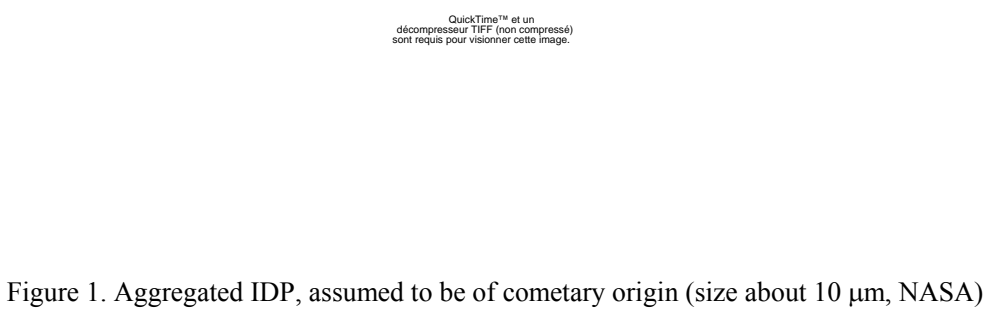

\section{Clues from remote observations}

\subsection{Spectroscopic observations}

While spectroscopic observations have provided a wealth of information on the 
composition of gaseous comae, their contribution to the understanding of the dust coma is more limited. However, spectra in the infrared near $10 \mu \mathrm{m}$ are of major interest. For Jupiter-family comets, they may reveal a weak silicate emission feature ${ }^{15}$; for long-period comets ${ }^{14}$, such as $1 \mathrm{P} / \mathrm{Halley}^{16}$ and C/1995 O1 Hale-Bopp ${ }^{17}$, which corresponds up to now to the strongest emission ever detected in a comet, they reveal strong silicate emission features. Analyses of such strong structured silicate emission features indicate the presence of both glassy and crystalline silicate components. Also, tiny crystalline water-ice grains have been suspected in the coma of C/1995 O1 Hale-Bopp ${ }^{18}$.

\subsection{Light scattering observations}

A key technique to reveal the properties of cometary dust particles from groundbased observations is to study the characteristics of the light they scatter and $\mathrm{emit}^{19}$. For randomly oriented particles, solar light scattered by an optically thin media is linearly polarized, with the electromagnetic wave predominantly oscillating perpendicular or parallel to the scattering plane. The linear polarization (thereafter polarization) is a ratio, by convention positive whenever the electromagnetic wave oscillates perpendicularly to the scattering plane. It only depends upon the phase angle $\alpha$ (between the light source and the observer, as seen from the scattering dust), the wavelength of the observations, and the properties of the dust particles, which may change with the location within the coma, with the activity of the comet (e.g. outburst, fragmentation), and with different classes of cometary dust (corresponding e.g. to a low or high maximum in polarization). The polarimetric phase curves obtained for cometary dust are smooth, with a shallow negative branch in the backscattering region up to about $20^{\circ}$, and a wide positive branch, with a maximum in the $90^{\circ}-100^{\circ}$ range. Once data are separated in different wavelength ranges, the dispersion on the positive branch is reduced, and different classes of comets (corresponding to a high or low maximum in polarization) can be noticed ${ }^{20}$. In the $25^{\circ}$ to $90^{\circ}$ phase angle range and in the visible domain, the polarization actually increases with the wavelength ${ }^{19,20}$.

The cometary polarimetric phase curves are similar to those of atmosphereless solar system bodies, and estimated to be typical of light scattering by irregular particles, with a size greater than the wavelength of the observations. Besides, the comets presenting a high maximum in polarization generally exhibit a strong silicate emission feature ${ }^{20}$. However, to interpret more accurately the polarization properties in terms of physical properties of the dust, both experimental and numerical simulations (with tentatively realistic particles) are needed.

\subsection{Interpretation through simulations}


We have developed a versatile model of cometary dust light scattering by compact grains and fluffy aggregates of grains, as well as an experimental programme with numerous levitating irregular dust samples.

The new numerical light scattering model, adapted from discrete dipole approximation, T-matrix and ray tracing methods, allows us to simulate the light scattered by a cloud of irregular particles, including for the first time spheroids and aggregates thereof ${ }^{21,22}$. Once polarization data are available on a sufficient range of phase angles and wavelengths for a given comet, this model gives clues to the bulk properties of the dust in the coma (i.e. size distribution, mass ratio between absorbing organics and less absorbing silicates) and to the structure of the particles.

From all data available for the extensively observed comet C/1995 O1 Hale-Bopp ${ }^{23,24}$, a very good $\mathrm{fit}^{21}$ is obtained by a root mean square minimization for data in the red $(670 \mathrm{~nm})$ and the green $(523 \mathrm{~nm})$ part of the visible spectrum (Fig. 2). The calculated phase curves present a negative branch below the inversion angle, and reproduce very well the data from $25^{\circ}$ to $50^{\circ}$. Moreover, for the same parameters, observations at other wavelengths, as shown in Fig. 2 for the near ultraviolet observations (from $344.8 \mathrm{~nm}$ to $365 \mathrm{~nm}$ ), are also accurately fitted.

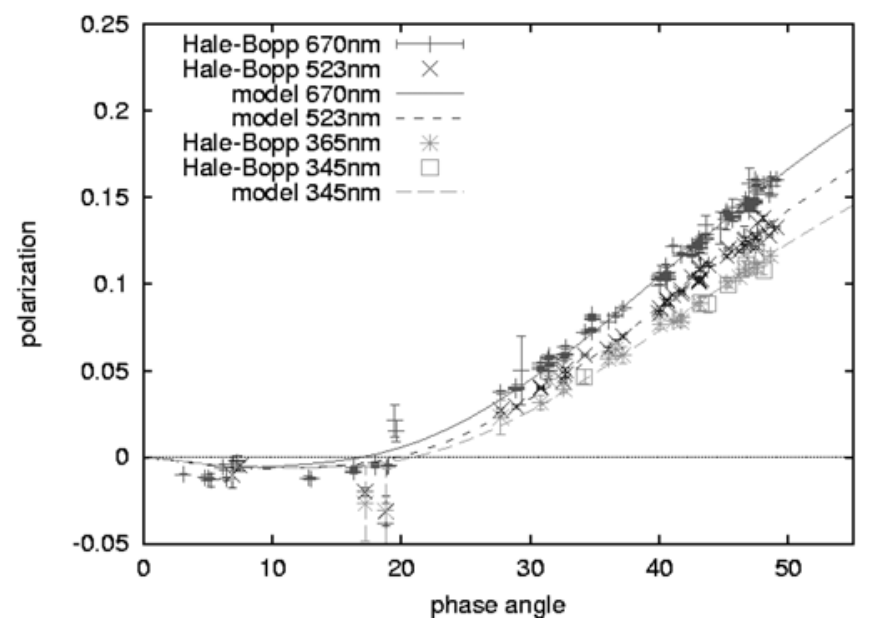

Figure 2. Phase curves derived from a numerical model fitting observational data for comet 1995 O1 Hale-Bopp in red (by $670 \mathrm{~nm}$ ) and green (by $523 \mathrm{~nm}$ ). The model, which requires silicates and organics, as well as compact grains and fluffy particles with sizes between 0.2 and $40 \mu \mathrm{m}$, also leads to a satisfactory fit of the near ultraviolet observations (by $345 \mathrm{~nm}$ ). 
These simulations therefore reproduce both the shape of the polarization phase curves and the quasi-linear variation of the polarization with the wavelength in the visible part of the spectrum with a limited range of free parameters, i.e. power-law index of the size distribution, equivalent radius of the smaller and larger dust particles, ratio in mass of non absorbing silicates to more absorbing organics. Both rather transparent silicates (with typically, $\mathrm{n} \approx 1.62+$ $\mathrm{i} 0.003$ at $550 \mathrm{~nm})$ and more absorbing organics $(\mathrm{n} \approx 1.88+\mathrm{i} 0.1$ at $550 \mathrm{~nm})$ are needed, as well as a significant proportion of aggregates (at least about 18\%). The size distribution power law index is of about -3 . Fluffy aggregates of tiny grains (with radii down to $0.1 \mu \mathrm{m}$ ) are needed, as well as compact grains (with equivalent radii up to $20 \mu \mathrm{m}$ ). The composition corresponds to $40 \%$ to $67 \%$ in mass of non absorbing silicates mixed with the complementary quantity of absorbing organics (33\% to $60 \%$ ), this range in percentage corresponding to the fact that part of the silicate grains may be embedded in absorbing organics mantles.

The same model applied to the interplanetary dust cloud (at 1.5 AU near the ecliptic plane, where a local inversion is feasible ${ }^{3}$ ) indicates the presence of both silicates and more absorbing particles ${ }^{22,25}$. Since the amount of aggregates is of about $20 \%$ in mass, the contribution of cometary dust particles is estimated to be of $20 \%$ at least. The observed decrease in polarization with decreasing solar distance is interpreted by a degradation of the organic material. It may be added that the presence of a still significant amount of aggregates (most likely of cometary origin) in the early interplanetary dust cloud is likely to have played a role in the delivery of prebiotic compounds to the surface of terrestrial planets during the late early bombardment phase ${ }^{26}$.

Finally, laboratory simulations of the light scattered by levitating cometary analog particles help to disentangle the different physical parameters ${ }^{27,28}$. Mixtures of fluffy silica and carbon aggregates present polarimetric phase curves similar to those obtained for comets, if the grains are in the 50 to $100 \mathrm{~nm}$ size-range and/or if some larger compact silica grains are added ${ }^{27}$. Fluffy chains are mandatory for the negative branch, and carbonaceous compounds for the positive spectral gradient. Recent measurements, with aggregates of submicronsized magnesio silicates, ferro silicates and carbon (expected to be the main components of cometary particles) and compact magnesio silicates, are even more comparable to those obtained by remote observations of dust in cometary comae $^{29}$.

\section{Evidence from recent space missions}

The Deep Impact mission ${ }^{30}$ to comet 9P/Tempel 1 has provided a confirmation of previous hypotheses on the fragility of comets' nuclei. From numerous 
observations before the impact, it was noticed that the nucleus suffered some outbursts typical of small fragmentations. Also its density could be estimated ${ }^{31}$, and found to be very low, of about $(350 \pm 250) \mathrm{kg} \mathrm{m}^{-3}$. As far as dust is concerned, the particles ejected by the impact were somehow different from those normally observed in a coma. Spectra in the near infrared suggest an increase in the amount of organics ${ }^{30}$ in the plume resulting from the impact, as well as the presence of very fined-grained water ice grains coming from the subsurface $^{32}$. However, spectroscopic observations could certainly not give unambiguous information about the mineralogy and crystallography of the dust particle in the coma and in the subsurface.

On the opposite, the Stardust Mission collected dust particles in the coma of periodic comet $81 \mathrm{P} /$ Wild 2 down to a miss distance of $236 \mathrm{~km}$, to allow detailed analysis in Earth laboratories, as well as fresh interstellar grains passing through the solar system (hence its name). The on-board instruments have confirmed the existence of dust fragmentation processes ${ }^{33}$ and the predominance of organic matter ${ }^{34}$. The dust was collected by the spacecraft, sufficiently far from the nucleus such that the largest particle captured was approximately 100 $\mu \mathrm{m}$ in size. On the return capsule, impacts ${ }^{35}$ on aluminium foils and tracks in the aerogel cells (Fig. 3) have revealed the presence of both cohesive and compact grains, as well as more friable particles (quite likely consisting of loosely bound aggregates) built of tiny strong grains.
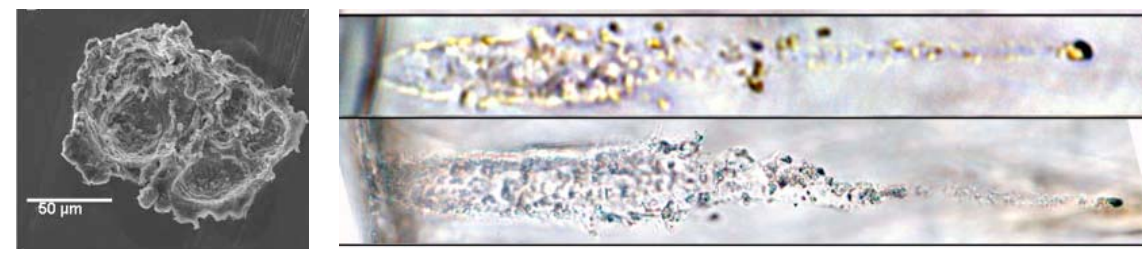

Figure 3. Evidence for dust aggregates in the cometary dust samples brought to Earth by Stardust. Left, impact of a loosely bound aggregate on an Al foil ${ }^{35}$. Right, two tracks (about $2 \mathrm{~mm}$ long) in aerogel cells, induced by friable particles built of tiny strong grains

More results should soon be available in a special issue of Meteoritics and Planetary Sciences (now in press). The power law of the size distribution of the above-mentioned particles has been found to be about -2.7 on the foils ${ }^{36}$. Analysis of the samples ${ }^{37,38}$ has demonstrated that Wild 2 contained an abundance of minerals with wildly different histories, from deuterium and ${ }^{15} \mathrm{~N}$ rich organics that must have formed at the very edge of the protosolar nebula, to refractory silicates and oxides that probably formed right up next to the early Sun. Virtually the entire early solar nebula appears to be represented among the returned particles. Presolar grains are also present among the Wild-2 samples, 
but appear to be rare. In general the captured grains appear to most closely resemble anhydrous chondritic interplanetary dust particles, though significant differences are apparent. The only hint of cometary aqueous alteration is the confirmed report of very rare $\mathrm{Mg}$-Fe carbonate grains in one sample, and the unconfirmed report of calcite in two other samples ${ }^{39,40}$. No phyllosilicates have been found. Given the extremely heterogeneous nature of Wild 2 grains one has to wonder whether the carbonates formed on Wild 2 or a precursor object, now destroyed.

\section{Perspectives and conclusions}

\subsection{Perspectives}

In the coming years, observations of the light scattering properties of cometary and interplanetary dust will be pursued, with numerous remote observations of active or bright comets (e.g. 17P/Holmes, C/2007 N3 Lulin). Besides, space missions such as Deep Impact at $85 \mathrm{P} /$ Boethin (DIXI) in 2008, Stardust at 9P/Tempel 1 (NEXT) in 2011, and PLANET-C during its cruise phase to Venus $^{39}$ will hopefully provide new data on cometary and interplanetary dust in a relatively near future. Simulations, including long duration dust samples agglomeration and light scattering measurements on board the ISS with the ICAPS experiment are also expected to provide precise data bases in the coming years.

In 2014-2015, the Rosetta rendezvous mission to the Jupiter family comet 67P/Churyumov-Gerasimenko should, for the fist time, provide a characterization of the nucleus and inner coma from near aphelion to perihelion, and a detailed study of the composition. After its successful launch in 2004, and Earth and Mars swing-bys in 2005-2006, Rosetta will flyby asteroids 2867 Steins in 2008 and 21 Lutetia in 2010, and reach Churyumov-Gerasimenko (size about $4 \mathrm{~km}$, active areas about $7 \%$, density in the $100-400 \mathrm{~kg} \mathrm{~m}^{-3}$ range) in early 2014 , before landing the Philae probe on the nucleus surface. Of special interest for the characterization of the dust particles properties should be GIADA ${ }^{40}$, MIDAS $^{41}$ and COSIMA ${ }^{42}$ experiments. Also, the CONSERT ${ }^{43}$ experiment should provide the first probing of the structure of the nucleus, together with information about its porosity. Later on, it may be expected that sample return missions (e.g. Marco Polo to a defunct comet nucleus or a primitive asteroid) will bring on Earth more samples of the refractory component of a comet.

\subsection{Conclusions}

It may be already concluded that cometary dust particles are irregular, that they consist of at least a bimodal population, with rather transparent (silicates) and 
rather absorbing (organic compounds) particles, that their morphology corresponds to both compact grains and fluffy aggregates, and that they significantly contribute to the replenishment of the interplanetary dust cloud. Ongoing studies will provide an optimization of the science return of the Rosetta rendezvous mission to a comet, which should contribute to a better accuracy of the comets formation models and a better understanding of the processes that have been taking place in the early solar system.

\section{Acknowledgments}

MZ was supported by the NASA Stardust discovery mission. JL was supported by a post-doctoral fellowship from the French Space Agency (CNES). The authors acknowledge fruitful discussions with E. Hadamcik, J.R. Renard and E. Burchell, and partial support from CNES.

\section{References}

1. K.H. Glassmeier, H. Boehnhardt, D. Koschny, E. Kührt and I. Richter, Space Sci. Rev. 128, 1 (2007)

2. E. Grün, B.A.S. Gustafson, S.F. Dermott and H. Fechtig (Eds), Interplanetary dust (Springer, Berlin, 2001)

3. A.C. Levasseur-Regourd, I. Mann, R. Dumont and M.S. Hanner, in: Interplanetary dust (Springer, Berlin, 2001)

4. M.C. Festou, H.U. Keller and H.A. Weaver (Eds), Comets II (Univ. of Arizona Press, Tucson, 2004)

5. S.J. Weidenschilling, in: Comets II (Univ. of Arizona Press, Tucson, 2004)

6. J. Blum et al., Phys. Rev. Lett. 85, 2426 (2000)

7. P. Ehrenfreund et al., Planet. Space Sci. 51, 473 (2003)

8. J. Kissel et al., Nature 321, 280 (1986)

9. M.N. Fomenkova, Space Sci. Rev. 90, 109 (1999)

10. A.C. Levasseur-Regourd, N. McBride, E. Hadamcik and M. Fulle. Astron. Astrophys. 348, 636 (1999)

11. M. Fulle, A.C. Levasseur-Regourd, N. McBride and E. Hadamcik, Astron. J. 119, 1968 (2000)

12. N. McBride, S. Green, A.C. Levasseur-Regourd, B. Goidet-Devel and J.B. Renard, Mon. Not. R. Astron. Soc. 289, 535 (1997)

13. F.M. Rietmeijer, Chemie der Erde 62, 1 (2002)

14. M.S. Hanner and J.P. Bradley, in: Comets II (University of Arizona Press, Tucson, 2004)

15. M.S. Hanner, D.K. Lynch, R.W. Russel, J.A. Hackwell and R. Kellogg, Icarus 124, 344 (1996) 
16. J.H. Bregman et al., Astron. Astrophys. 187, 616 (1987)

17. J. Crovisier et al., Science 275, 1904 (1997)

18. E. Lellouch et al., Astron. Astrophys. 339, L9 (1998)

19. L. Kolokolova, M.S. Hanner, A.C. Levasseur-Regourd and B.A.S. Gustafson, in: Comets II (University of Arizona Press, Tucson (2004)

20. A.C. Levasseur-Regourd, E. Hadamcik and J.B. Renard. Astron. Astrophys. 313, 327 (1996)

21. J. Lasue and A.C. Levasseur-Regourd. J. Quant. Spectros. Radiat. Transfer 100, 220 (2006)

22. A.C. Levasseur-Regourd, T. Mukai, J. Lasue and Y. Okada, Planet. Space Sci. 55, 1010 (2007)

23. E. Hadamcik, A.C. Levasseur-Regourd and J.B. Renard, Earth Moon Planets 78, 365-371, 1997

24. E. Hadamcik and A.C. Levasseur-Regourd, Astron. Astrophys. 403, 757 (2003)

25. J. Lasue, A.C. Levasseur-Regourd, N. Fray and H. Cottin, Astron. Astrophys. 473, 641 (2007)

26. A.C. Levasseur-Regourd, J. Lasue and E. Desvoivres, Origin of Life and Evolution of Biosphere 36, 507 (2006)

27. E. Hadamcik, J.B. Renard, A.C. Levasseur-Regourd and J. Lasue, J. Quant. Spectros. Radiat. Transfer 100, 143 (2006)

28. H. Volten, O. Munoz, J.W. Hovenier, F.J.M. Rietmeijer, J.A. Nuth, L.B.F. Waters and W.J. van der Zande. Astron. Astrophys. 470, 377 (2007)

29. E. Hadamcik, J.B. Renard, F.J.M. Rietmeijer, A.C. Levasseur-Regourd, H.G.M. Hill, J.M. Karner and J.A. Nuth. Icarus 190, 660 (2007)

30. M.F. A'Hearn et al., Science 310, 258 (2005)

31. M.F. A'Hearn and M.R. Combi, Icarus 187, 1 (2007)

32. J.M. Sunshine et al., Icarus 190, 284 (2007)

33. A.J. Tuzzolino et al., Science 304, 1776 (2004)

34. J. Kissel, F.R. Krueger, J. Silen and B.C. Clark, Science 304, 1774 (2004)

35. F. Hörz et al., Science 314, 1716 (2006)

36. M.J. Burchell et al., Meteoritics and planetary science, In press (2007)

37. M. Zolensky et al., Science 314, 1735 (2006)

38. M. Zolensky et al., Meteoritics and planetary sciences, In press (2007)

39. T. Mikouchi et al., Lunar And Planetary Science XXXVIII, 1956 (2007)

40. S. Wirick et al., Lunar And Planetary Science XXXVIII, 1534 (2007)

41. M. Ueno et al., AOGS 58-PS-A0560 (2007)

42. L. Colangeli et al., Space Sci. Rev. 128, 803 (2007)

43. W. Riedler et al., Space Sci. Rev. 128, 869 (2007)

44. J. Kissel et al., Space Sci. Rev. 128, 823 (2007)

45. W. Kofman et al., Space Sci. Rev. 128, 413 (2007) 
